\title{
Aptamer conjugated silver nanoparticles for the detection of interleukin 6
}

\author{
Andrea K. Locke ${ }^{1}$, Nicole Norwood ${ }^{1}$, Haley L. Marks ${ }^{1}$, Monika Schechinger ${ }^{1}$, George W. Jackson²,3, Duncan Graham ${ }^{4}$, \\ Gerard L. Coté ${ }^{1}$ \\ ${ }^{1}$ Department of Biomedical Engineering, Texas A\&M University, College Station, Texas, United States \\ ${ }^{2}$ BioTex, Inc., Houston, Texas, United States \\ ${ }^{3}$ Base Pair Biotechnologies, Inc., Pearland, Texas, United States \\ ${ }^{4}$ Department of Pure and Applied Chemistry, University of Strathclyde, Glasgow, Scotland
}

\begin{abstract}
The controlled assembly of plasmonic nanoparticles by a molecular binding event has emerged as a simple yet sensitive methodology for protein detection. Metallic nanoparticles (NPs) coated with functionalized aptamers can be utilized as biosensors by monitoring changes in particle optical properties, such as the LSPR shift and enhancement of the SERS spectra, in the presence of a target protein. Herein we test this method using two modified aptamers selected for the protein biomarker interleukin 6 , an indicator of the dengue fever virus and other diseases including certain types of cancers, diabetes, and even arthritis. IL6 works by inducing an immunological response within the body that can be either anti-inflammatory or pro-inflammatory. The results show that the average hydrodynamic diameter of the NPs as measured by Dynamic Light Scattering was $\sim 42$ $\mathrm{nm}$. After conjugation of the aptamers, the peak absorbance of the AgNPs shifted from 404 to $408 \mathrm{~nm}$ indicating a surface modification of the NPs due to the presence of the aptamer. Lastly, preliminary results were obtained showing an increase in SERS intensity occurs when the IL-6 protein was introduced to the conjugate solution but the assay will still need to be optimized in order for it to be able to monitor varying concentration changes within and across the desired range.
\end{abstract}

Keywords: Surface enhance Raman spectroscopy (SERS), aptamer, interleukin 6, plasmonic nanoparticles, binding assay, protein detection

\section{INTRODUCTION}

Due to its high sensitivity surface enhanced Raman spectroscopy (SERS) is a desirable optical modality that can be used to monitor molecular-molecular interactions. The detection of protein-molecular binding via SERS analysis of functionalized nanoparticles has been extensively studied over the years. ${ }^{1}$ SERS provides a controlled system where the change in Raman enhancement from the introduction of a Raman reporter molecule (RRM) can be manipulated to track changes in molecular binding based on either a "turn-on" or "turn-off" scheme. This work utilizes the "turn-on" approach where aptamer conjugated colloidal silver nanoparticles aggregates under controlled conditions to form a sandwich pair in the presence of the aptamer-specific protein. The assembly of these particles in a sandwich formation results in localized "hot spots" of electric fields surrounding the RRM. Therefore, as more of these sandwich pairs are formed in the presence of increasing concentrations of binding protein, the number of scattering events should increase resulting in an increase in the SERS spectral intensity.

For the purpose of this study, the desired protein to be detected is Interleukin 6 (IL6). IL6 is a protein present in the blood that results from a number of alignments and can act as a pro-inflammatory or anti-inflammatory agent. ${ }^{2}$ Most commonly it is secreted by the immune system in response to trauma, as in the case of introduction of foreign types of bacteria or viruses. IL6 can also be secreted from high levels of strain typically endured by the muscles. ${ }^{3}$ Recently, IL6 has been shown to be secreted in the body to create an immune response due to the presence of certain types of diseases such as: diabetes, lupus, certain types of cancers, arthritis, and most importantly for this study, the dengue virus. ${ }^{3}$ 
Contraction of the dengue virus leads to the development of a disease commonly referred to as Dengue Fever (DF). While DF is most commonly characterized by a high fever, body aches, and rashes, it can become deadly as it progresses and patients undergo shock. ${ }^{3}$ A portable and easy to use device can allow doctors to detect and act to avoid the onset of shock followed by death.

\section{MATERIALS \& METHODS}

\subsection{Materials and Instrumentation}

The modified IL6 aptamer77: 5'-[aptamer77][HEG] 3 THIOL-3' and aptamer83: 5'- [aptamer83][HEG] 3 THIOL-3' were synthesized by Base Pair Biotechnologies Inc. (U.S.A). Malachite green isothiocyanate (MGITC) reporter dye was purchased from Life Tecnologies ${ }^{\circledR}$ (U.S.A). All other reagents were obtained from Sigma Aldrich (USA). A Tecan microplate reader was used to measure absorbance on the synthesized particles with a scan range of 240-600 nm. The hydrodynamic diameter of the nanoparticles were measured via Dynamic Light Scattering on a Zetasizer Nano ZS90 (Malvern, U.K.). SERS measurements were collected using a Raman spectroscopy instrument (Thermo Scientific, U.S.A) fitted with a piezo-driven XYZ scan stage. All samples were probed using a laser wavelength of $532 \mathrm{~nm}$ and 900 grooves per $\mathrm{mm}$ grating.

\subsection{Colloid Synthesis of Silver colloid (AgNP)}

AgNP was synthesized using the method reported by Leopold and Lendl. ${ }^{4}$ Briefly, 1 mL hydroxylamine hydrochloride $(150 \mathrm{mM})$ was added to $89 \mu \mathrm{L}$ of $\mathrm{NaOH}(3.33 \mathrm{mM})$ vortexing at $6000 \mathrm{rpm}$. Next, $10 \mathrm{~mL}$ Silver nitrate $\left(\mathrm{AgNO}_{3}\right)$ solution $(10 \mathrm{mM})$ was added drop-wise and allowed to stir for 15 minutes at room temperature. Dynamic Light Scattering measurements were then taken to determine the average particle diameter of the silver solution. The concentration was the particles was determined via UV-Vis spectroscopy using an extinction coefficient of $2.87 \times 10^{10} \mathrm{M}^{-1} \mathrm{~cm}^{-1}$ at its peak wavelength of $\sim 404 \mathrm{~nm} .^{5}$

\subsection{Aptamer/RRM AgNP Conjugation}

Aptamer-77 and aptamer-83 were added and eventually conjugated to separate batches of the previously synthesized AgNP. Prior to conjugation, the aptamers were suspended in $60 \mathrm{mM}$ phosphate buffer ( $\mathrm{pH} 8.5$ ) to achieve a stock solution of $100 \mu \mathrm{M}$ as recommended by the distributor. In order for conjugation to occur, the stock solutions of aptamer-77 and aptamer-83 were treated with $20 \mathrm{mM}$ of tris (2-carboxyethyl) phosphine (TCEP) at room temperature. The treatment of TCEP allowed the aptamers to be capable of interacting and binding to the AgNPs by the reducing their disulfide bonds. After 1 hour, the solutions of aptamer-77 and aptamer-83 were purified to remove residual TCEP, via a 3kDa Nanoseps ${ }^{\circledR}$ desalting centrifuge column. Both solutions underwent this purification method a total of three times before ultimately being resuspended in $1 \mathrm{~mL}$ of PBS $(0.1 \mathrm{M}$ with 10 $\mathrm{mM} \mathrm{MgCl} 2, \mathrm{pH} 7.4)$. To achieve optimal binding, the aptamers were submerged in a water bath $\left(\sim 90^{\circ} \mathrm{C}\right)$ in the presence of $10 \mathrm{mM} \mathrm{MgCl}_{2}$ for a total of 5 minutes to aid the aptamers to fold into their appropriate tertiary structures.

The AgNPs were then functionalized with the aptamers using a modified method developed by Zhang et al. ${ }^{6}$ First, aptamer-77 was added to $1 \mathrm{~mL}$ of silver colloid at a molar ratio of 5000:1 and was left shaking for 1 hour to create optimal conditions for the reaction to occur. Next, in three $20 \mu \mathrm{L}$ aliquots, $250 \mathrm{mM}$ citrate $\mathrm{HCl}$ buffer $(\mathrm{pH}$ 2.9) was added to the aptamer-77 conjugated AgNP solution and left to mix for an additional 20 minutes. The particles were then washed three times to remove any unconjugated aptamer by centrifuging the particles at $7000 \mathrm{rpm}$ for 5 minutes. Each time, the supernatant was replaced with $1 \mathrm{~mL}$ of PBS $\left(0.1 \mathrm{M}\right.$ with $10 \mathrm{mM} \mathrm{MgCl}_{2}$, $\mathrm{pH}$ 7.4). This method was repeated to synthesize a separate $1 \mathrm{~mL}$ batch of aptamer-83 conjugated AgNPs. 
The absorbance was determined for both solutions of conjugated aptamer-77 and aptamer-83 AgNPs. By using Beer's Law, the concentration of AgNPs conjugated with aptamers was calculated using the appropriate absorbance readings. The solution of AgNPs conjugated with aptamer-83 consistently showed a higher concentration. Based on this information, the solution containing AgNPs conjugated with aptamer-83 was chosen to be tagged with a Raman reporter molecule. No further modification was performed to the solution containing the conjugated apatamer-77 AgNPs. The conjugated apatamer-77 AgNPs were instead suspended and stored in $0.1 \mathrm{M}$ PBS buffer solution (with $10 \mathrm{mM} \mathrm{MgCl}_{2}$ at $\mathrm{pH}$ 7.4).

After conjugation and analysis of the apatamer-83 AgNPs, $10 \mu \mathrm{M}$ of the Raman reporter molecule, malachite green isothiocyanate (MGITC) in methanol, was added at a molar ratio of 500:1 (MGITC to aptamer-83 AgNPs). The solution of conjugated aptamer-83 AgNPs and MGITC was mixed for 1 hour to ensure adequate attachment of the Raman reporter molecule was achieved. The solution of conjugated aptamer-83 AgNPs with Raman reporter (aptamer-83-MGITC AgNPs) was then washed via centrifugation and stored in $0.1 \mathrm{M}$ PBS buffer solution (with $10 \mathrm{mM} \mathrm{MgCl}_{2}$ at $\mathrm{pH}$ 7.4).

\subsection{SERS analysis}

Equal volumes containing $30 \mathrm{pM}$ of the conjugated aptamer nanoparticles were obtained from the solutions previously synthesized. The assays were tested in $30 \mu \mathrm{L}$ wells $(10 \mu \mathrm{L}$ of aptamer-77 AgNPs, $10 \mu \mathrm{L}$ of aptamer83-MGITC AgNPs, and $10 \mu \mathrm{L}$ of target protein) by analyzing the SERS spectra. Prior to testing the assay in its entirety, both solutions of conjugated aptamers were tested separately. To account for dilution, the aptamers were tested in $30 \mu \mathrm{L}$ wells containing $20 \mu \mathrm{L}$ of Nuclease-Free Deionized Water and $10 \mu \mathrm{L}$ of conjugated aptamer AgNPs. This was done to ensure the RRM had successfully bonded to the solution of aptamer-83 AgNPs which resulted in a high absorbance spectra.

The assay was tested using $10 \mu \mathrm{L}$ of aptamer-77 AgNPs, $10 \mu \mathrm{L}$ of aptamer-83-MGITC AgNPs, and $10 \mu \mathrm{L}$ of the IL-6 protein $(1 \mu \mathrm{M})$. An increase in the SERS intensity would indicate functionality of the aptamer conjugated assay.

\section{RESULTS \& DISCUSSION}

The average hydrodynamic diameter of the AgNPs as measured by Dynamic Light Scattering was $\sim 42 \mathrm{~nm}$. After conjugation of the aptamers, the peak absorbance of the AgNPs shifted from 404 to $408 \mathrm{~nm}$ indicating a surface modification of the NPs due to the presence of the aptamer.

To test the assay functionality the SERS intensity of MGITC was monitored as the IL-6 protein was introduced to the conjugate solution containing equal volumes of aptamer-77 AgNPs and aptamer-83/RRM AgNPs. The preliminary result shown in Figure 1 shows an increase in SERS intensity when the IL-6 protein was introduced to the conjugate solution. 


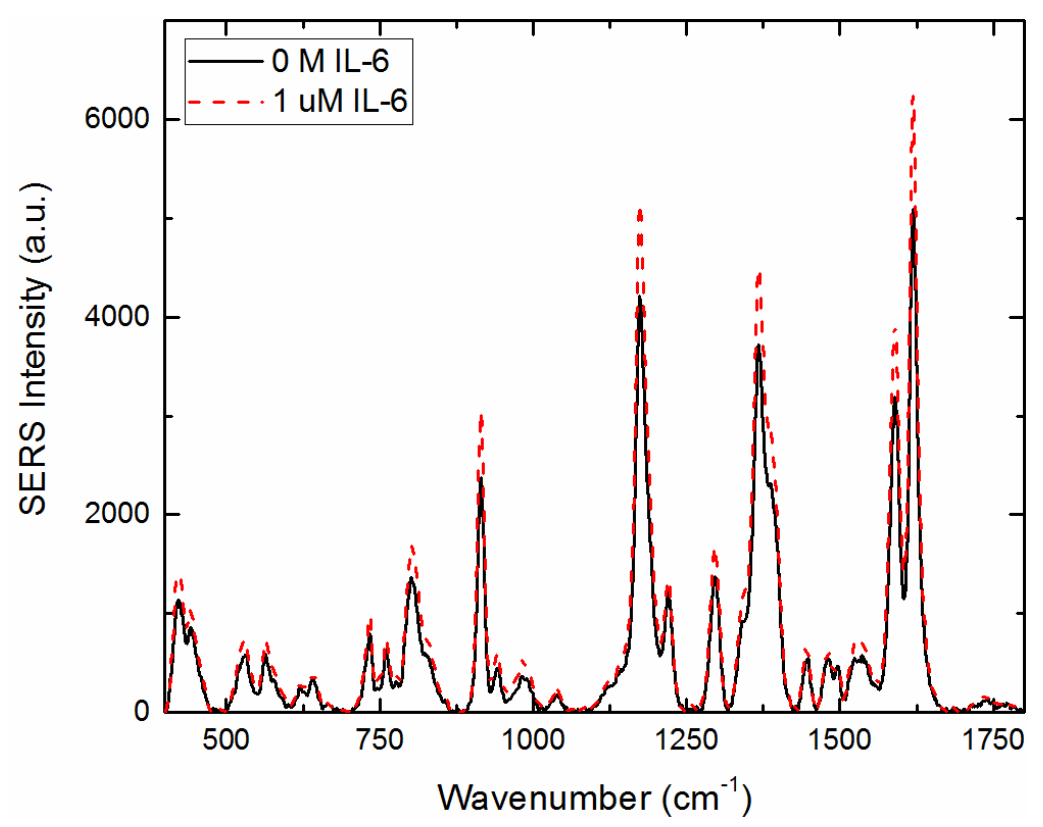

Figure 1. SERS spectra of aptamer conjugated AgNPs in the presence of IL-6 protein

\section{CONCLUSION \& FUTURE WORKS}

In this work, we have designed a "turn on" SERS sandwich assay for the detection of the IL-6 protein. Although there was a slight increase in intensity when the protein was introduced this assay will still need to be optimized in order for it to be able to monitor varying concentration changes within and across the desired range.

\section{REFERENCES}

1. Graham, Duncan, and Royston Goodacre. "Chemical and bioanalytical applications of surface enhanced Raman scattering spectroscopy." Chemical Society Reviews 37.5 (2008): 883-884.

2. Alonzi, Tonino, et al. "Interleukin 6 is required for the development of collagen-induced arthritis." The Journal of experimental medicine 187.4 (1998): 461-468.

3. Jufferie, M; van der Meer, GM; Hack, CE; Haasnoot, K; Sutrayo; Veerman, AJP; Thijs, LG; Inflammatory Mediators in Dengue Virus Infection in Children: Interleukin-8 and Its Relationship to Neutrophil Degranulation. Infect Immun. 2000 Feb; 68(2): 702-707.

4. Leopold, N. \& Lendl, B. A new method for fast preparation of highly surface-enhanced Raman scattering (SERS) active silver colloids at room temperature by reduction of silver nitrate with hydroxylamine hydrochloride. The Journal of Physical Chemistry B 107, 5723-5727 (2003).

5. Yguerabide, J. \& Yguerabide, E.E. Light-Scattering Submicroscopic Particles as Highly Fluorescent Analogs and Their Use as Tracer Labels in Clinical and Biological Applications: I. Theory. Analytical Biochemistry 262, 137-156 (1998).

6. X. Zhang, M. R. Servos, and J. Liu, "Fast pH-assisted functionalization of silver nanoparticles with monothiolated DNA," Chem. Commun., vol. 48, no. 81, pp. 10114-10116, (2012). 